\title{
Hydrogen-Induced High-Temperature Superconductivity in Two-Dimensional Materials: The Example of Hydrogenated Monolayer $\mathrm{MgB}_{2}$
}

\author{
J. Bekaert, ${ }^{1, *}$ M. Petrov, ${ }^{1}$ A. Aperis, ${ }^{2}$ P. M. Oppeneer, ${ }^{2}$ and M. V. Milošević ${ }^{1, \dagger}$ \\ ${ }^{1}$ Department of Physics, University of Antwerp, Groenenborgerlaan 171, B-2020 Antwerp, Belgium \\ ${ }^{2}$ Department of Physics and Astronomy, Uppsala University, P.O. Box 516, SE-751 20 Uppsala, Sweden
}

(Received 10 February 2019; revised manuscript received 23 April 2019; published 13 August 2019)

\begin{abstract}
Hydrogen-based compounds under ultrahigh pressure, such as the polyhydrides $\mathrm{H}_{3} \mathrm{~S}$ and $\mathrm{LaH}_{10}$, superconduct through the conventional electron-phonon coupling mechanism to attain the record critical temperatures known to date. Here we exploit the intrinsic advantages of hydrogen to strongly enhance phonon-mediated superconductivity in a completely different system, namely, a two-dimensional material with hydrogen adatoms. We find that van Hove singularities in the electronic structure, originating from atomiclike hydrogen states, lead to a strong increase of the electronic density of states at the Fermi level, and thus of the electron-phonon coupling. Additionally, the emergence of high-frequency hydrogen-related phonon modes in this system boosts the electron-phonon coupling further. As a concrete example, we demonstrate the effect of hydrogen adatoms on the superconducting properties of monolayer $\mathrm{MgB}_{2}$, by solving the fully anisotropic Eliashberg equations, in conjunction with a first-principles description of the electronic and vibrational states, and their coupling. We show that hydrogenation leads to a high critical temperature of $67 \mathrm{~K}$, which can be boosted to over $100 \mathrm{~K}$ by biaxial tensile strain.
\end{abstract}

DOI: 10.1103/PhysRevLett.123.077001

In seminal work of 1968, Ashcroft showed that dense metallic hydrogen, if ever produced, could be a hightemperature superconductor [1]. The main reason would be its very high Debye temperature, as a result of its minimal mass, enabling very strong phonon-mediated superconducting pairing according to the Bardeen-CooperSchrieffer (BCS) theory. Subsequent detailed firstprinciples studies yielded critical temperature $\left(T_{c}\right)$ values up to $242 \mathrm{~K}$, along with descriptions of the multiband nature of superconductivity [2], and the role of phonon anharmonicities [3]. However, as creating metallic hydrogen requires immense pressures of $\sim 400 \mathrm{GPa}[4,5]$, a confirmation of high- $T_{c}$ superconductivity in pure hydrogen systems is still pending [6].

Instead, in search of hydrogen-induced high-temperature superconductivity, most researchers have turned to polyhydrides, compounds with a large hydrogen content, but also containing at least one other chemical element. The latter enables stabilizing the structure under lower applied pressure compared to metallic hydrogen itself. Notably, the chalcogen hydrides display experimentally proven high- $T_{c}$ superconductivity, e.g., $T_{c}=203 \mathrm{~K}$ in $\mathrm{H}_{3} \mathrm{~S}$ [7], supplemented by comparable theoretical predictions for $\mathrm{H}-\mathrm{Te}$ compounds [8]. The record $T_{c}$ 's among all currently known superconductors are held by the rare-earth hydrides, notably $\mathrm{LaH}_{10}$, with $T_{c}$ of 250-260 K [9,10], and there is also a theoretical prediction of an even higher $T_{c}=303 \mathrm{~K}$ in $\mathrm{YH}_{10}$ [11].

In this work we demonstrate a different approach to establish high- $T_{c}$ superconductivity based on hydrogen, namely, by adding hydrogen adatoms to two-dimensional superconductors [12-14], exploiting the changes in the electronic and vibrational properties that hydrogen induces. Such 2D superconductivity has been realized in recent years in very diverse ultrathin materials, ranging from atomically thin elemental metal films ( $\mathrm{Pb}$, In, etc.) [15-17], over monolayers (MLs) of unconventional superconductors [18], monolayer FeSe with high $T_{c}$ up to $100 \mathrm{~K}[19,20]$, to truly 2D atomic sheets such as doped graphene [21-24] and transition metal dichalcogenides [25-30].

As an illustrative example, we consider hydrogenation of a monolayer magnesium diboride $\left(\mathrm{MgB}_{2}\right)$. Our main motivation to focus on this material is that it hosts distinct surface states [31], thus allowing for adatoms to be adsorbed at its surface. Further, ML $\mathrm{MgB}_{2}$ structurally resembles lithium-doped graphene, another $2 \mathrm{D}$ superconductor of recent interest [22-24]. Finally, ML $\mathrm{MgB}_{2}$ harbors three different types of electronic states at the Fermi level [32] that may be prone to rich hybridization with adatoms, and a readily significant electron-phonon (e-ph) coupling that may be boosted by vibrational contributions of the adatoms. As we will show, hydrogen indeed contributes to all those aspects of superconductivity in hydrogenated $\mathrm{ML} \mathrm{MgB}_{2}\left(\mathrm{H}-\mathrm{MgB}_{2}\right)$.

As the first step, we have determined the equilibrium structure of the $\mathrm{H}-\mathrm{MgB}_{2}$ system, based on density functional theory (DFT), as implemented in ABINIT [33,34] [all computational details are provided in the Supplemental Material (SM) [35]]. To this end we have considered several possible high-symmetry positions of the $\mathrm{H}$ adatoms 
within the $\mathrm{MgB}_{2}$ unit cell, at the side of the magnesium plane, and have calculated the resulting phonon dispersions. In this search, as detailed in the SM [38], we obtained only one dynamically stable structure, with one hydrogen adatom per unit cell. This structure, shown in Fig. 1(a), is characterized by hydrogen positions corresponding to one of the two sublattices of the underlying boron honeycomb structure, resulting in tricoordination of the adjacent magnesium atoms. As for thermodynamic stability, we find that the hydrogenation reaction lowers the internal energy of the $\mathrm{H}-\mathrm{MgB}_{2}$ system (calculated within DFT). As a result, decomposing the structure of Fig. 1(a) into monolayer $\mathrm{MgB}_{2}$ and $\mathrm{H}_{2}$ gas is not energetically favorable. Conversely, any additional amount of hydrogen beyond one per $\mathrm{MgB}_{2}$ unit leads to an increase in internal energy, suggesting that $\mathrm{H}$ would be removed from the monolayer in the form of $\mathrm{H}_{2}$ molecules. During the hydrogenation process, at lower adatom concentrations, hydrogen adatoms at only one of the two sublattice sites are again energetically favored, which promotes efficient growth of the ground state structure with uniform hydrogen coverage.

The corresponding electronic band structure of $\mathrm{H}-\mathrm{MgB}_{2}$ is displayed in Fig. 1(b) [35]. Around the Fermi level $\left(E_{F}\right)$

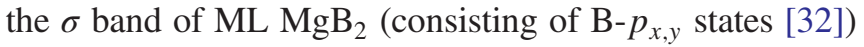
persists, while the characteristic surface band stemming from the free $\mathrm{Mg}$ surface is eliminated by hydrogenation. Interestingly, the $\mathrm{H}-s$ state forms a hybridized bond with the B- $p_{z}$ states on the other side of the stack, as can be seen through the wave function in Fig. 1(c). Thus, a new electronic band emerges as a result of hydrogenation, indicated as the $\pi-\mathrm{H} s$ state, in view of its hybridized band character. The resulting 2D Fermi surface consists of two $\sigma$

(a)

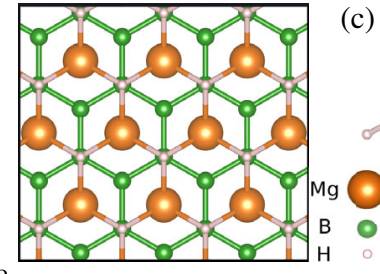

(c)

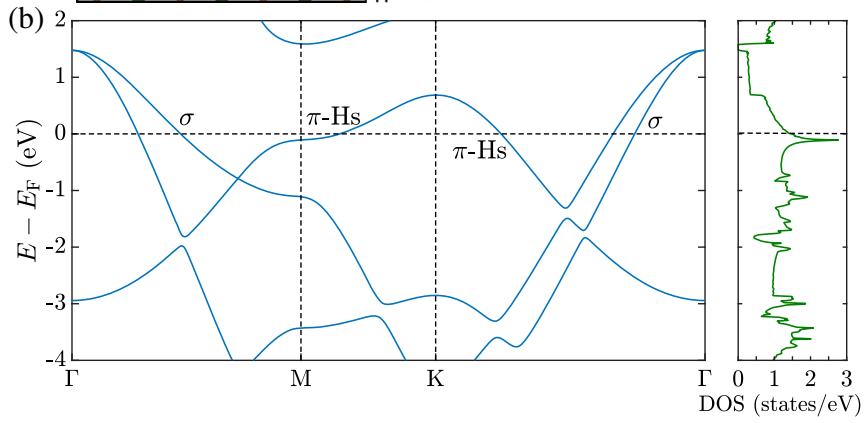

FIG. 1. Structural and electronic properties of $\mathrm{H}_{-} \mathrm{MgB}_{2}$. (a) Top view of the crystal structure. (b) The electronic band structure and density of states (DOS). The $\sigma$ and $\pi$-Hs bands crossing $E_{F}$ are also indicated. (c) The norm of the wave function of the $\pi$-Hs state at $E_{F}$ obtained along the path $\Gamma-K$. sheets around $\Gamma$ and the $\pi$-Hs state around $\mathrm{K}$, as shown in Fig. 3(a).

The hybridized $\pi-\mathrm{H} s$ state in $\mathrm{H}-\mathrm{MgB}_{2}$ carries more density of states (DOS) than its pure $\pi$ counterpart in $\mathrm{ML} \mathrm{MgB}_{2}$, which is beneficial for superconductivity. The total DOS at $E_{F}$ is $\sim 50 \%$ larger in $\mathrm{H}-\mathrm{MgB}_{2}$ compared with $\mathrm{ML} \mathrm{MgB}_{2}$ (1.41 states/eV versus 0.96 states/eV, respectively). The DOS enhancement stems mainly from the flat dispersion of the $\pi$-Hs state around point $M$ [cf., Fig. 1(b)]. This results in a van Hove singularity in the DOS just below $E_{F}$. We generally expect hydrogenation to contribute to a flat dispersion due to the atomiclike $\mathrm{H}-s$ state with very limited overlap [cf., Fig. 1(c)]. Since the van Hove singularity occurs merely $109 \mathrm{meV}$ below $E_{F}$, the superconducting properties of $\mathrm{H}-\mathrm{MgB}_{2}$ can be further (and significantly) enhanced with limited $p$ doping so as to maximize the DOS at $E_{F}$. This effect bears a resemblance to the case of twisted bilayer graphene, where superconductivity appears due to a flatband interlayer state, albeit that there the flat dispersion extends throughout the whole Brillouin zone and results from interlayer correlations [40].

As a next step, we determined the vibrational properties and the electron-phonon (e-ph) coupling in $\mathrm{H}_{-} \mathrm{MgB}_{2}$ using density functional perturbation theory calculations [41-43]. The resulting phonon band structure, shown in Fig. 2(a), proves the dynamical stability of the $\mathrm{H}-\mathrm{MgB}_{2}$ structure. In this phonon band structure, the mode- $(\nu)$ and momentum(q) dependent $e$-ph coupling $\lambda^{\nu}(\mathbf{q})$ is also indicated. The largest $\lambda^{\nu}(\mathbf{q})$ values occur for $\mathbf{q}=0$ (i.e., at $\Gamma$ ). The three modes with maximal coupling, depicted in Fig. 2(b), are (I) a shear mode of the $\mathrm{B}$ plane, on one hand, and the $\mathrm{Mg}$ and $\mathrm{H}$ planes, on the other hand, (II) a B-B in-plane stretching mode where the $\mathrm{H}$ atoms move in phase with the $\mathrm{B}$ atoms of the other sublattice, and (III) $\mathrm{Mg}$ and $\mathrm{H}$ moving out of phase in the out-of-plane direction. Note that in all three modes $\mathrm{H}$ atoms are involved. Mode II is similar to the strongly coupling $\mathrm{C}$-C stretching mode in superconducting $p$-doped graphane, where the $\mathrm{H}$ atoms move along [44]. Therefore, apart from the electronic hybridization described above, there is also a hybridization of the vibrational modes of $\mathrm{H}$ with those of the other atoms. In general, $\mathrm{H}$ atoms are prone to vibrationally hybridize as a result of their minimal mass. This is corroborated by the phonon DOS shown in Fig. 2(a), where a significant overlap of the $\mathrm{H}$ and $\mathrm{B}$ contributions can be observed. Finally, in the rightmost panel of Fig. 2(a), the isotropically averaged Eliashberg spectral function $\alpha^{2} F$ [45] of the frequency-dependent $e$-ph coupling is displayed. The resulting isotropic $e$-ph coupling constant attains a high value of $\lambda_{\text {iso }}=1.46$ in ML H-MgB 2 , compared with $\lambda_{\text {iso }}=$ 0.68 in nonhydrogenated $\mathrm{ML} \mathrm{MgB}_{2}$ [32]. This strong enhancement of $\lambda$ due to hydrogenation is a combined effect of a higher electronic DOS and emerging strongcoupling phonon modes, both stemming from hybridization with electronic and vibrational states of hydrogen, as discussed above. 

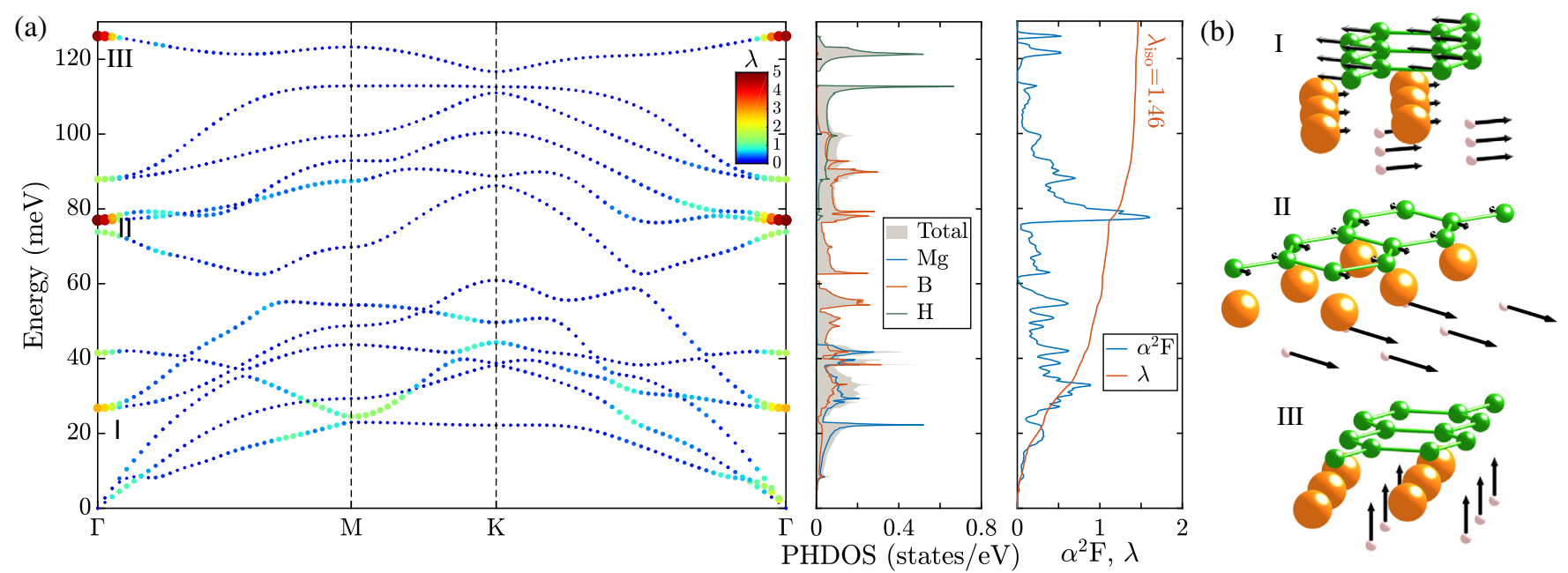

FIG. 2. Phonons and electron-phonon interaction in $\mathrm{H}_{-} \mathrm{MgB}_{2}$. (a) Phonon band structure (where both the colors and the dot sizes indicate the strength of the $e$-ph coupling $\lambda$ ), phonon density of states (PHDOS, including the atom-resolved contributions), and isotropic Eliashberg function $\alpha^{2} F$ (plus the $e$-ph coupling constant $\lambda$ ). (b) Phonon modes with the strongest $e$-ph coupling, labeled as indicated in (a).

To accurately calculate the influence of hydrogenation on the superconducting properties of $\mathrm{ML} \mathrm{H}-\mathrm{MgB}_{2}$, we used our first-principles results for electrons, phonons, and their coupling field as input to solve the fully anisotropic Migdal-Eliashberg equations as implemented in the Uppsala superconductivity code (UPPSC) [31,32,46-50] (see SM for computational details [35]). Notwithstanding the high phonon frequencies of $\mathrm{H}-\mathrm{MgB}_{2}$ due to hydrogen [cf., Fig. 2(a)], Migdal's approximation [45] is valid in $\mathrm{H}-\mathrm{MgB}_{2}$, as the electronic characteristic energy scale still dominates (the ratio of the maximum phonon energy $E_{\mathrm{ph}}$ and the electronic bandwidth $E_{e}$-limited by the $\pi$-Hs stateamounts to $E_{\mathrm{ph}} / E_{e}=0.18 \ll 1$ ).

By solving the fully anisotropic formalism we obtain the superconducting gap $\Delta(\mathbf{k})$ as a function of electron momentum $\mathbf{k}$ (specifically the Fermi wave vectors). The results shown in Fig. 3(a) indicate strong Cooper pairing $\left(\Delta / T_{c}=2.24\right.$ as opposed to the BCS ratio of 1.76) in $\mathrm{ML} \mathrm{H}-\mathrm{MgB}_{2}$, with $\Delta$ values in the range $11.5-13 \mathrm{meV}$ (at a low temperature of $1 \mathrm{~K}$ ). $\Delta$ in the range $11.5-12.2 \mathrm{meV}$ stems predominantly from the $\mathrm{H}-s$ state, whereas the highest $\Delta$ values are due to the $\sigma$ bands. Nevertheless, the distribution $\rho(\Delta)$ does not present separated domes for each contributing state, i.e., multigap behavior, as is the case in $\mathrm{ML} \mathrm{MgB}_{2}$ [32]. We attribute the mixing of the pairing strengths upon hydrogenation to the emergence of significant interband coupling, through $\lambda^{\nu}(\mathbf{q})$ with nonzero $\mathbf{q}$ [cf., Fig. 2(a)]. Such interband scattering is particularly strong between the two $\sigma$ states and the $\pi$-H $s$ state (see SM for further information [35]).

Figure 3(b) shows the temperature evolution of $\rho(\Delta)$, obtained from solving the Eliashberg equations for each temperature separately. This gives $T_{c}=67 \mathrm{~K}$, the temperature where $\Delta$ vanishes. Since $\mathrm{ML} \mathrm{MgB}_{2}$ has $T_{c}=20 \mathrm{~K}$
[32], hydrogenation has strongly enhanced the superconducting pairing strength and the corresponding $T_{c}$. In order to assess the contributions of the different chemical elements in $\mathrm{H}-\mathrm{MgB}_{2}$, we also investigated the isotope effect [51]. To this end we renormalized the phonon frequencies according to the isotope masses, and subsequently solved again the Eliashberg equations. Apart from the $\mathrm{B}$ isotope effect that is essentially unchanged compared to pure $\mathrm{MgB}_{2}$ (see SM for details [52]), we found a quite strong $\mathrm{H}$ isotope effect. Namely, $T_{c}$ decreases from 67 to $63 \mathrm{~K}$ when hydrogen is replaced with deuterium, and
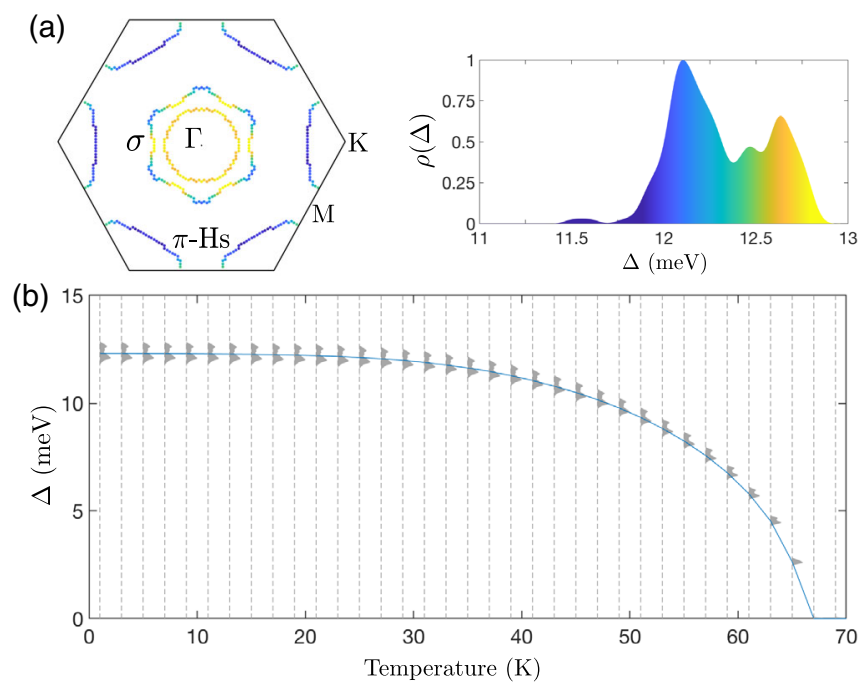

FIG. 3. Superconducting properties of $\mathrm{H}-\mathrm{MgB}_{2}$, calculated with fully anisotropic Eliashberg theory. (a) The superconducting gap spectrum on the Fermi surface, calculated at $1 \mathrm{~K}$, and the corresponding distribution $\rho(\Delta)$ with the color code. (b) The evolution of $\rho(\Delta)$ with temperature, yielding $T_{c}=67 \mathrm{~K}$. 
further down to $59 \mathrm{~K}$ for tritium, supporting again the crucial role of $\mathrm{H}$ in the high-temperature superconductivity of $\mathrm{H}-\mathrm{MgB}_{2}$.

In all of these calculations we have described the Coulomb interaction between the Cooper-pair electrons using a MorelAnderson pseudopotential [55] of $\mu^{*}=0.13$. We note, however, that our main result, the strong enhancement of superconductivity due to hydrogenation, persists even for an increased Coulomb interaction, as detailed in the SM [35].

At this point, we recall that the properties of $2 \mathrm{D}$ materials are relatively easily manipulated by lattice deformation or strain, due to a substrate or by exerting a force on a suspended monolayer [32,56-58]. To check the corresponding effect on superconductivity in $\mathrm{H}-\mathrm{MgB}_{2}$, we applied in-plane biaxial strain, where the hexagonal symmetry of the lattice is preserved. We carried out firstprinciples calculations with strain in the range $-5 \%$ (compressive) to 5\% (tensile) strain. We found that hydrogen greatly stiffens the material due to its intrinsically high phonon frequencies, so that $\mathrm{H}-\mathrm{MgB}_{2}$ cannot be compressively strained beyond $-1 \%$, yet the phonon spectra proved stable for tensile strain up to $5 \%$. The change in the Eliashberg function when the in-plane lattice parameter is increased by $5 \%$, as calculated from first principles, is shown in Fig. 4(a). Because of such applied tensile strain the phonon frequencies lower overall, and in addition, some of the main peak amplitudes increase. This increase stems from changes in the electronic structure, as with tensile strain the van Hove singularity in the electronic DOS moves closer to $E_{F}$ (as shown in the SM [35]), such that the Fermi sheets of the $\pi$-Hs band increase, as shown in Fig. 4(b) [cf., Fig. 3(a)]. These combined changes in $\alpha^{2} F$ lead to a strong boost of the $e$-ph coupling, to $\lambda_{\text {iso }}=2.03$ (compared with 1.46 in the unstrained case), since $\lambda$ relates to the integral over frequencies of $\alpha^{2} F(\omega) / \omega$.

On the basis of the obtained first-principles results, we carried out anisotropic Eliashberg calculations, to visualize the changes in the superconducting properties. The obtained gap spectrum $\Delta(\mathbf{k})$ and corresponding distribution $\rho(\Delta)$ in the case of 5\% tensile strain displayed in Fig. 4(b) reveal a great boost in $\Delta$ with respect to the unstrained case, to the range of 20 to $22 \mathrm{meV}$, as a result of the enhanced $e$-ph coupling. Such high $\Delta$ values are characteristic of hightemperature superconductivity. In Fig. 4(c) we show the temperature evolution of $\Delta$, from which we obtain $T_{c}=103 \mathrm{~K}$. In the inset we show the $T_{c}$ values for all studied strain values, yielding an almost linear dependence. By using substrates with a slightly larger lattice constant (e.g., $\mathrm{Si}_{1+x} \mathrm{C}_{1-x}$ or $\mathrm{Al}_{x} \mathrm{Ga}_{1-x} \mathrm{~N}$ alloys, with a lattice constant tunable by $x$ [59]), tensile strain can thus be applied to obtain desired $\Delta$ values and to increase $T_{c}$ of $\mathrm{H}-\mathrm{MgB}_{2}$ above $100 \mathrm{~K}$.

In conclusion, our study establishes hydrogen as an ideal adatomic candidate to enhance the superconducting properties of 2D materials, and to boost their critical temperatures to the high- $T_{c}$ range. We showed that hydrogen adatoms (a)
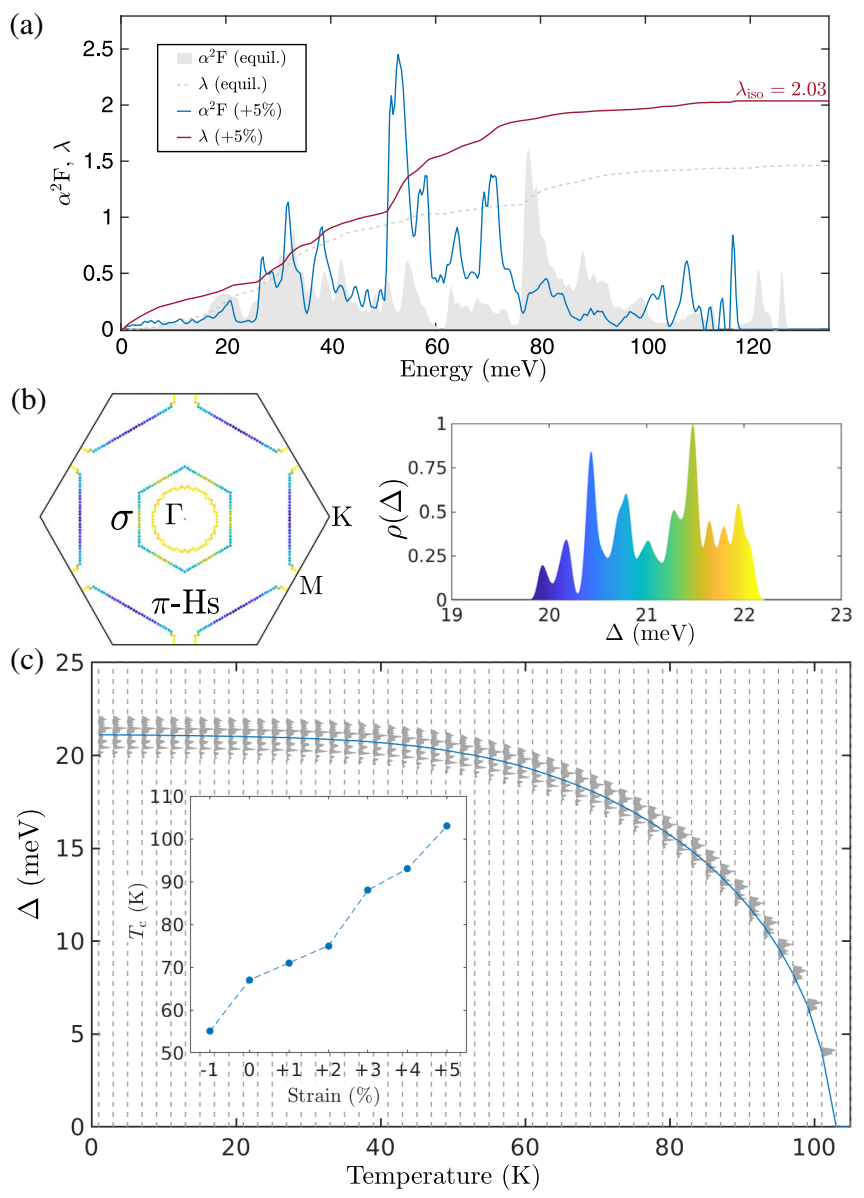

FIG. 4. Superconducting properties of $\mathrm{H}-\mathrm{MgB}_{2}$ under the influence of strain. (a) Isotropic Eliashberg function $\alpha^{2} F$ and electron-phonon coupling constant $\lambda$ for applied $5 \%$ tensile strain. The data for the nonstrained case are shown by the gray shadow for comparison. (b) The superconducting gap spectrum on the Fermi surface, from fully anisotropic calculations for applied 5\% tensile strain, and the corresponding color-coded $\rho(\Delta)$. (c) The evolution of $\rho(\Delta)$ with temperature for $5 \%$ tensile strain, yielding $T_{c}=103 \mathrm{~K}$. The inset shows $T_{c}$ as a function of strain.

adsorbed on a 2D superconductor open new electronphonon coupling channels at high frequencies, and that hydrogen's electronic and vibrational states are very prone to hybridization, thereby enhancing preexisting Cooperpairing channels. Furthermore, a major advantage of these hybridized electronic states is their vanishing dispersion at certain parts of the Brillouin zone, owing to hydrogen's atomiclike $s$ orbital, introducing van Hove singularities in the electronic density of states. Occurring in the vicinity of the Fermi level, these strongly enhance the electron-phonon coupling, and thus the $T_{c}$.

As a concrete example, we showed that the above reasons yield a high $T_{c}$ of $67 \mathrm{~K}$ in a hydrogenated monolayer of magnesium diboride, that can be boosted above $100 \mathrm{~K}$ by merely $5 \%$ of tensile biaxial strain. This proves that hydrogenation of a $2 \mathrm{D}$ material can indeed induce strong electron-phonon coupling and high- $T_{c}$ 
superconductivity, as exploited in bulk hydride compounds with record $T_{c}$ 's to date $[9,10]$, yet without the need to apply excessively high pressures that hamper practical applications.

This work was supported by TOPBOF-UAntwerp, Research Foundation-Flanders (FWO), the Swedish Research Council (VR), the Röntgen-Ångström Cluster, and the EU-COST Action CA16218. J. B. acknowledges support of a postdoctoral fellowship of the FWO. The computational resources and services used for the firstprinciples calculations in this work were provided by the VSC (Flemish Supercomputer Center), funded by the FWO and the Flemish Government-department EWI. Eliashberg theory calculations were supported through the Swedish National Infrastructure for Computing (SNIC). We would also like to acknowledge useful discussions with Bart Partoens, Jacques Tempère, and Matthieu Verstraete.

J. B. and M. P. contributed equally to this work.

*jonas.bekaert@uantwerpen.be †milorad.milosevic@uantwerpen.be

[1] N. W. Ashcroft, Phys. Rev. Lett. 21, 1748 (1968).

[2] P. Cudazzo, G. Profeta, A. Sanna, A. Floris, A. Continenza, S. Massidda, and E. K. U. Gross, Phys. Rev. Lett. 100, 257001 (2008).

[3] I. Errea, M. Calandra, C. J. Pickard, J. Nelson, R. J. Needs, Y. Li, H. Liu, Y. Zhang, Y. Ma, and F. Mauri, Phys. Rev. Lett. 114, 157004 (2015).

[4] S. Azadi, B. Monserrat, W. M. C. Foulkes, and R. J. Needs, Phys. Rev. Lett. 112, 165501 (2014).

[5] J. McMinis, R. C. Clay, D. Lee, and M. A. Morales, Phys. Rev. Lett. 114, 105305 (2015).

[6] R. P. Dias and I. F. Silvera, Science 355, 715 (2017).

[7] A. P. Drozdov, M. I. Eremets, I. A. Troyan, V. Ksenofontov, and S. I. Shylin, Nature (London) 525, 73 (2015).

[8] X. Zhong, H. Wang, J. Zhang, H. Liu, S. Zhang, H.-F. Song, G. Yang, L. Zhang, and Y. Ma, Phys. Rev. Lett. 116, 057002 (2016).

[9] M. Somayazulu, M. Ahart, A. K. Mishra, Z. M. Geballe, M. Baldini, Y. Meng, V. V. Struzhkin, and R. J. Hemley, Phys. Rev. Lett. 122, 027001 (2019).

[10] A. P. Drozdov, P. P. Kong, V. S. Minkov, S. P. Besedin, M. A. Kuzovnikov, S. Mozaffari, L. Balicas, F. F. Balakirev, D. E. Graf, V. B. Prakapenka, E. Greenberg, D. A. Knyazev, M. Tkacz, and M. I. Eremets, Nature (London) 569, 528 (2019).

[11] F. Peng, Y. Sun, C. J. Pickard, R. J. Needs, Q. Wu, and Y. Ma, Phys. Rev. Lett. 119, 107001 (2017).

[12] Y. Saito, T. Nojima, and Y. Iwasa, Nat. Rev. Mater. 2, 16094 (2017).

[13] T. Uchihashi, Supercond. Sci. Technol. 30, 013002 (2017).

[14] C. Brun, T. Cren, and D. Roditchev, Supercond. Sci. Technol. 30, 013003 (2017).
[15] T. Zhang, P. Cheng, W. Li, Y. Sun, G. Wang, X. Zhu, K. He, L. Wang, X. Ma, X. Chen, Y. Wang, Y. Liu, H. Lin, J. Jia, and Q. Xue, Nat. Phys. 6, 104 (2010).

[16] J. Noffsinger and M. L. Cohen, Solid State Commun. 151, 421 (2011).

[17] M. Yamada, T. Hirahara, and S. Hasegawa, Phys. Rev. Lett. 110, 237001 (2013).

[18] A. T. Bollinger, G. Dubuis, J. Yoon, D. Pavuna, J. Misewich, and I. Bozovic, Nature (London) 472, 458 (2011).

[19] R. Peng, X. P. Shen, X. Xie, H. C. Xu, S. Y. Tan, M. Xia, T. Zhang, H. Y. Cao, X. G. Gong, J. P. Hu, B. P. Xie, and D. L. Feng, Phys. Rev. Lett. 112, 107001 (2014).

[20] J. Ge, Z. Liu, C. Liu, C. Gao, D. Qian, Q. Xue, Y. Liu, and J. Jia, Nat. Mater. 14, 285 (2015).

[21] K. Kanetani, K. Sugawara, T. Sato, R. Shimizu, K. Iwaya, T. Hitosugi, and T. Takahashi, Proc. Natl. Acad. Sci. U.S.A. 109, 19610 (2012).

[22] G. Profeta, M. Calandra, and F. Mauri, Nat. Phys. 8, 131 (2012).

[23] B. M. Ludbrook, G. Levy, P. Nigge, M. Zonno, M. Schneider, D. J. Dvorak, C. N. Veenstra, S. Zhdanovich, D. Wong, P. Dosanjh, C. Straßer, A. Stöhr, S. Forti, C. R. Ast, U. Starke, and A. Damascelli, Proc. Natl. Acad. Sci. U.S.A. 112, 11795 (2015).

[24] J.-J. Zheng and E. R. Margine, Phys. Rev. B 94, 064509 (2016).

[25] X. Xi, Z. Wang, W. Zhao, J.-H. Park, K. T. Law, H. Berger, L. Forro, J. Shan, and K. F. Mak, Nat. Phys. 12, 139 (2016).

[26] M. M. Ugeda, A. J. Bradley, Y. Zhang, S. Onishi, Y. Chen, W. Ruan, C. Ojeda-Aristizabal, H. Ryu, M. T. Edmonds, H.-Z. Tsai, A. Riss, S.-K. Mo, D. Lee, A. Zettl, Z. Hussain, Z.-X. Shen, and M. F. Crommie, Nat. Phys. 12, 92 (2016).

[27] J. Pan, C. Guo, C. Song, X. Lai, H. Li, W. Zhao, H. Zhang, G. Mu, K. Bu, T. Lin, X. Xie, M. Chen, and F. Huang, J. Am. Chem. Soc. 139, 4623 (2017).

[28] Y. Yang, S. Fang, V. Fatemi, J. Ruhman, E. NavarroMoratalla, K. Watanabe, T. Taniguchi, E. Kaxiras, and P. Jarillo-Herrero, Phys. Rev. B 98, 035203 (2018).

[29] J. T. Ye, Y. J. Zhang, R. Akashi, M. S. Bahramy, R. Arita, and Y. Iwasa, Science 338, 1193 (2012).

[30] Y. Saito, Y. Nakamura, M. S. Bahramy, Y. Kohama, J. Ye, Y. Kasahara, Y. Nakagawa, M. Onga, M. Tokunaga, T. Nojima, Y. Yanase, and Y. Iwasa, Nat. Phys. 12, 144 (2016).

[31] J. Bekaert, L. Bignardi, A. Aperis, P. van Abswoude, C. Mattevi, S. Gorovikov, L. Petaccia, A. Goldoni, B. Partoens, P. M. Oppeneer, F. M. Peeters, M. V. Milosevic, P. Rudolf, and C. Cepek, Sci. Rep. 7, 14458 (2017).

[32] J. Bekaert, A. Aperis, B. Partoens, P. M. Oppeneer, and M. V. Milošević, Phys. Rev. B 96, 094510 (2017).

[33] X. Gonze et al., Comput. Phys. Commun. 180, 2582 (2009).

[34] D. R. Hamann, Phys. Rev. B 88, 085117 (2013).

[35] See Supplemental Material at http://link.aps.org/ supplemental/10.1103/PhysRevLett.123.077001 for the corresponding charge analysis, which includes Refs. [36,37].

[36] R. Bader, Atoms in Molecules-A Quantum Theory (Oxford University Press, Oxford, 1990).

[37] W. Tang, E. Sanville, and G. Henkelman, J. Phys. Condens. Matter 21, 084204 (2009).

[38] This includes Ref. [39] relating to the space group properties. 
[39] International Tables for Crystallography, edited by T. Hahn (Springer, New York, 2005), Vol. A.

[40] Y. Cao, V. Fatemi, S. Fang, K. Watanabe, T. Taniguchi, E. Kaxiras, and P. Jarillo-Herrero, Nature (London) 556, 43 (2018).

[41] S. Y. Savrasov, Phys. Rev. Lett. 69, 2819 (1992).

[42] S. Y. Savrasov and D. Y. Savrasov, Phys. Rev. B 54, 16487 (1996).

[43] F. Giustino, Rev. Mod. Phys. 89, 015003 (2017).

[44] G. Savini, A. C. Ferrari, and F. Giustino, Phys. Rev. Lett. 105, 037002 (2010).

[45] P. B. Allen and B. Mitrović, in Theory of Superconducting $T_{c}$, edited by H. Ehrenreich, F. Seitz, and D. Turnbull, Solid State Physics Vol. 37 (Academic Press, New York, 1983), pp. 1-92.

[46] G. M. Eliashberg, J. Exp. Theor. Phys. 11, 696 (1960).

[47] A. Aperis, P. Maldonado, and P. M. Oppeneer, Phys. Rev. B 92, 054516 (2015).

[48] J. Bekaert, S. Vercauteren, A. Aperis, L. Komendová, R. Prozorov, B. Partoens, and M. V. Milošević, Phys. Rev. B 94, 144506 (2016).
[49] J. Bekaert, A. Aperis, B. Partoens, P. M. Oppeneer, and M. V. Milošević, Phys. Rev. B 97, 014503 (2018).

[50] A. Aperis and P. M. Oppeneer, Phys. Rev. B 97, 060501(R) (2018).

[51] The results shown in Fig. 3(a) have been obtained with weighted averages of the isotope masses, according to their natural occurrence.

[52] This includes Refs. [53,54] for the isotope effect in bulk $\mathrm{MgB}_{2}$.

[53] S. L. Bud'ko, G. Lapertot, C. Petrovic, C. E. Cunningham, N. Anderson, and P. C. Canfield, Phys. Rev. Lett. 86, 1877 (2001).

[54] D. G. Hinks, H. Claus, and J. D. Jorgensen, Nature (London) 411, 457 (2001).

[55] P. Morel and P. W. Anderson, Phys. Rev. 125, 1263 (1962).

[56] J. Pešić, R. Gajić, K. Hingerl, and M. Belić, Europhys. Lett. 108, 67005 (2014).

[57] C. Si, Z. Liu, W. Duan, and F. Liu, Phys. Rev. Lett. 111, 196802 (2013).

[58] C. Si, Z. Sun, and F. Liu, Nanoscale 8, 3207 (2016).

[59] J.-C. Zheng and Y. Zhu, Phys. Rev. B 73, 024509 (2006). 